\title{
MUDANÇAS NO DESENVOLVIMENTO TEMPORAL DA FORÇA EM MULHERES DE DIFERENTES FAIXAS ETÁRIAS
}

CHANGES IN TEMPORAL DEVELOPMENT OF THE FORCE IN DIFFERENT AGE GROUPS OF WOMEN

CAMBIOS EN EL DESARROLLO TEMPORAL DE LA FUERZA EN MUJERES DE DIFERENTES

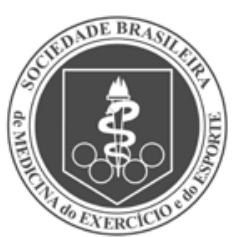

Artigo Original GRUPOS DE EDAD

\author{
Josária Ferraz Amaral ${ }^{1}$ \\ (Educador Físico) \\ Eliane Aparecida de Castro² \\ (Educador Físico) \\ Leonice Aparecida Doimo² \\ (Educador Físico) \\ Marcus Vinicius da Silva' \\ (Educador Físico) \\ José Marques Novo Júnior ${ }^{3}$ \\ (Educador Físico)
}

1. Universidade Federal de Juiz de Fora, Juiz de Fora, MG, Brasil.

2. Universidade Federal de Viçosa, Viçosa, MG, Brasil.

3. Universidade Federal de São

Carlos, São Carlos, SP, Brasil.

\section{Correspondência:}

Rua Orestes Pereira, no 50 apt 102, Juiz de Fora, MG, Brasil. 36037-300. josaria_ferraz@hotmail.com

\section{RESUMO}

Introdução: redução na força e na velocidade da contração muscular são duas das maiores causas de debilidade funcional em idosos. Objetivo: analisar as mudanças relacionadas à idade no desenvolvimento temporal da força de preensão manual e dos extensores do joelho em mulheres. Métodos: a amostra foi constituída por 52 mulheres subdivididas em três grupos (JO. $n=24,25,2 \pm 3,7$ anos; MI. $n=13,58 \pm 4,2$ anos; ID. $n=15,71,3 \pm 3,9$ anos). Os testes realizados foram o de preensão manual e o de extensão do joelho. Foram executadas três tentativas de esforço isométrico máximo em cada teste, e a curva com o valor máximo de força foi selecionada para análise por meio de um algoritmo desenvolvido em linguagem Matlab ${ }^{\circledR}$. A massa livre de gordura (MLG) foi avaliada por meio da absortometria radiológica de dupla energia. Resultados: foram observados entre os grupos valores semelhantes de força ( $p=0,831)$ e MLG do membro superior $(p=0,207)$. A taxa de desenvolvimento da força (TDF) foi menor nos intervalos iniciais da contração muscular (0-50 e 0-100ms) nos grupos MI ( $p=0,005$ e $p=0,000$, para $50 \mathrm{~ms}$ e $100 \mathrm{~ms}$, respectivamente) e ID ( $p=0,003$ e $p=0,000$, para $50 \mathrm{~ms}$ e $100 \mathrm{~ms}$, respectivamente). A MLG e a força do membro inferior foram menores nos grupos $\mathrm{MI}(\mathrm{p}=0,006, p=0,014$, respectivamente) e ID ( $p<0,000$, $p=0,000$, respectivamente) em comparação ao JO. Já a TDF dos extensores do joelho foi semelhante no grupo MI $(p>0,05)$, porém, menor no grupo ID $(p<0,05)$ em comparação ao JO, em todos os intervalos analisados. Conclusão: os resultados deste estudo sugerem que os grupos musculares dos membros superiores e inferiores possam ser afetados em diferentes magnitudes pelo envelhecimento. As diferenças entre os membros podem estar relacionadas à quantidade e qualidade das atividades físicas realizadas.

Palavras-chave: envelhecimento, força muscular, força da mão.

\section{ABSTRACT}

Introduction: reduction in strength and reduction in speed of muscle contraction are the two major causes of functional impairment in the elderly. Objective: to examine age-related changes in the temporal development of the force of the handgrip and knee extensors in women. Methods: the sample consisted of 52 women subdivided in three groups (JO. $n=24,25.2 \pm 3.7$ years; MI. $n=13,58 \pm 4.2$ years; ID. $n=15,71.3 \pm 3.9$ years). Tests of handgrip and knee extension were performed. Three attempts of maximum isometric effort were performed in each test, and the curve with the maximum strength value was selected for analysis by means of an algorithm developed using MATLAB ${ }^{\circledR}$. The fat free mass (FFM) was assessed by Dual-energy X-ray absorptiometry. Results: we observed similar values in strength $(p=0.831)$ and FFM ( $p=0.207)$ of the upper limb. The rate of the force development (RFD) was lower in the initial periods of muscle contraction (0-50 and 0-100ms) in the Ml group ( $p=0.005$ and $p=0.000$ for $50 \mathrm{~ms}$ and 100ms, respectively) and ID ( $p=0.003 p=0.000$ for $50 \mathrm{~ms}$ and $100 \mathrm{~ms}$, respectively). The FFM and lower limb strength were lower in the MI group ( $p=0.006, p=0.014$, respectively) and ID ( $p<0.000, p=0.000$, respectively) compared to the JO. Already, the RFD of the knee extensors was similar in the Ml group ( $p>0.05)$, but lower in the ID group $(p<0.05)$ compared to the $\mathrm{JO}$ in all analyzed intervals. Conclusion: the results of this study demonstrate that the muscle groups of the upper and lower limbs are affected in different magnitudes by the aging process. The differences between limbs may be related to the quantity and quality of physical activities.

Keywords: aging, muscle strength, hand strength.

\section{RESUMEN}

Introducción: reducción en la fuerza y en la velocidad de la contracción muscular son dos de las mayores causas de debilidad funcional en ancianos. Objetivo: analizar los cambios relacionados a la edad en el desarrollo temporal de la fuerza de aprehensión manual y de los extensores de la rodilla en mujeres. Métodos: la muestra fue constituida por 52 mujeres subdivididas en tres grupos (JO. $n=24,25,2 \pm 3,7$ años; MI. $n=13,58 \pm 4,2$ años; ID. $n=15,71,3 \pm 3,9$ años). Los tests realizados fueron el de aprehensión manual y el de extensión de la rodilla. Fueron ejecutadas tres tentativas de esfuerzo isométrico máximo en cada test, y la curva con el valor máximo de fuerza fue seleccionada para análisis por medio de un algoritmo desarrollado en lenguaje Matlab ${ }^{\circledR}$. La masa libre de grasa (MLG) fue evaluada por medio de la absortometría radiológica de doble energía. Resultados: fueron observados entre los grupos 
valores semejantes de fuerza $(p=0,831)$ y MLG del miembro superior $(p=0,207)$. La tasa de desarrollo de la fuerza (TDF) fue menor en los intervalos iniciales de la contracción muscular (0-50 y 0-100ms) en los grupos MI ( $p=0,005$ y $p=0,000$, para $50 \mathrm{~ms}$ y $100 \mathrm{~ms}$, respectivamente) e ID ( $p=0,003$ y $p=0,000$, para $50 \mathrm{~ms}$ y $100 \mathrm{~ms}$, respectivamente). La $M L G$ y fuerza del miembro inferior fue menor en los grupos $M I$ ( $p=0,006, p=0,014$, respectivamente) e ID ( $p<0,000$, $p=0,000$, respectivamente) en comparación al JO. Ya la TDF de los extensores de la rodilla fue semejante en el grupo MI ( $p>0,05)$, aunque menor en el grupo ID $(p<0,05)$ en comparación al JO, en todos los intervalos analizados. Conclusión: los resultados de este estudio sugieren que los grupos musculares de los miembros superiores e inferiores puedan ser afectados en diferentes magnitudes por el envejecimiento. Las diferencias entre los miembros pueden estar relacionadas a la cantidad y calidad de las actividades físicas realizadas.

Palabras clave: envejecimiento, fuerza muscular, fuerza de la mano.

\section{INTRODUÇÃO}

O declínio na força muscular voluntária máxima com o envelhecimento está relacionado a várias alterações nos sistemas musculoesquelético e nervoso ${ }^{1}$. Essa redução associada ao declínio da velocidade da contração muscular são as maiores causas de debilidade funcional em idosos ${ }^{2}$. Adicionalmente, estudos têm demonstrado que a habilidade de gerar força rapidamente pode ser mais funcionalmente relevante do que a capacidade de gerar força máxima ${ }^{3,4}$, uma vez que muitas das atividades da vida diária são caracterizadas pelo limitado tempo de desenvolvimento de força (50-200ms), o qual é consideravelmente menor do que o necessário para alcançar a força máxima ( $\geq 300 \mathrm{~ms})^{5}$.

Ademais, as alterações neuromusculares relacionadas ao envelhecimento não se manifestam de forma homogênea em todos os grupos musculares dos membros superiores e inferiores. Estudos demonstram que os membros inferiores são mais afetados pelo processo de envelhecimento do que os membros superiores ${ }^{6,7}$. Segundo Lynch et al. ${ }^{7}$, a massa muscular e a força dos membros inferiores parecem ser mais afetadas pelo processo de envelhecimento do que os membros superiores, principalmente devido ao fato de a redução do nível de atividade física afetar em maior extensão os membros inferiores. Além disso, indivíduos com fraqueza dos membros inferiores tendem a compensar os movimentos desses membros com outros músculos, tais como os do braço e, dessa forma, esse estresse regular nos membros superiores poderia contribuir para a manutenção da massa muscular e força desses membros ${ }^{6}$.

Na avaliação da capacidade de desenvolver força rapidamente, a taxa de desenvolvimento da força (TDF), definida como a inclinação da curva força-tempo ( $\triangle$ força/ $\Delta$ tempo) obtida durante ação muscular isométrica, tem sido utilizada em jovens e idosos, tanto na avaliação dos membros superiores ${ }^{3,8}$ quanto na dos membros inferiores ${ }^{4,9,10}$. Contudo, poucos estudos analisaram o comportamento da TDF dos membros superiores e inferiores na mesma amostra, e, além disso, a maioria dos trabalhos são realizados, em amostras compostas por ambos os gêneros ou somente por homens, dificultando a extrapolação desses resultados para as mulheres. O objetivo deste estudo foi analisar as mudanças relacionadas à idade no desenvolvimento temporal da força de preensão manual e dos extensores do joelho em mulheres.

\section{MATERIAIS E MÉTODOS}

A amostra foi constituída por 52 mulheres estratificadas de acordo com a idade cronológica em grupo jovem (JO) com 24 voluntárias (25,2 $\pm 3,7$ anos), grupo meia idade (MI) com 13 voluntárias (58 $\pm 4,2$ anos)

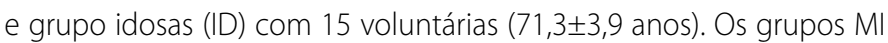
e ID foram compostos por mulheres participantes de um grupo de socialização realizado pelo Departamento de Educação Física da Universidade Federal de Viçosa, MG, Brasil. O grupo JO foi composto por estudantes de diferentes cursos da mesma instituição.
Foram excluídas do estudo mulheres portadoras de doença musculoesquelética, lesões, traumatismos ou que tenham sido submetidas à intervenção cirúrgica nos membros avaliados. A pesquisa foi realizada após a assinatura do Termo de Consentimento Livre e Esclarecido aprovado pelo Comitê de Ética em Pesquisa em Seres Humanos da Universidade Federal de Juiz de Fora (UFJF), Juiz de Fora, MG, Brasil, protocolo CEP/UFJF 2451.191.2011, parecer n. ${ }^{\circ}$ 201/2011.

As avaliações da força muscular dos membros superior e inferior foram realizadas indiretamente por meio dos testes de preensão manual e extensão do joelho. Tais testes foram executados, respectivamente, por meio da utilização do transdutor de força acoplado à empunhadura modificada por Novo Júnior ${ }^{1}$ e da célula de carga (tensiômetro), ambos da marca EMG System do Brasil. Os instrumentos possuíam conexão com um condicionador de sinais, com frequência de amostragem de $1024 \mathrm{~Hz}$ por canal.

Em cada avaliação, foram executadas três tentativas de esforço isométrico máximo, por 6 segundos, com intervalo de dois minutos entre elas, a fim de se evitar a instalação de fadiga muscular acumulada. A força muscular, tanto de extensão do joelho quanto de preensão manual, foi analisada no membro dominante.

Para os testes de preensão manual, padronizou-se a posição segundo orientações da $\mathrm{ASHT}^{12}$, na qual o sujeito permanece confortavelmente sentado, ombro aduzido e sem rotação, o cotovelo fletido a 90 graus e em posição neutra, posição do punho variando entre 0 a 30 graus de extensão. As voluntárias, dessa forma, envolviam a empunhadura com a mão, enquanto o dinamômetro era suportado pelo examinador. O tamanho da empunhadura do dinamômetro foi selecionado conforme Amaral et al. ${ }^{13}$.

Na avaliação da força dos extensores do joelho, as voluntárias foram orientadas a permanecer sentadas com as mãos apoiadas em suportes localizados nas laterais da cadeira, com o tronco ereto e ajustado pelo encosto, de forma a permitir um ângulo de 90 graus de flexão da articulação do quadril. A articulação do joelho do membro inferior dominante foi posicionada em 90 graus de flexão. A célula de carga que compõe o tensiômetro foi fixada em uma barra e, por meio de um cabo de aço, posicionada paralelamente ao chão com a outra extremidade acoplada a uma caneleira fixada no tornozelo do membro dominante da voluntária. Anteriormente ao início de cada avaliação, foi permitido às voluntárias um período de familiarização com o equipamento e com o protocolo de teste requerido.

Em ambos os testes, foram fornecidas orientações verbais de incentivo por parte do avaliador. Todas as voluntárias foram instruídas a executar a força máxima no menor tempo possível após o comando verbal para iniciar o teste, bem como a manter esse nível de força até que fosse fornecido o comando para relaxar.

Para as medidas de massa corporal e estatura foi utilizada, respectivamente, uma balança marca Filizola (Brasil) com precisão de 
100 gramas e um estadiômetro Sanny escalonado em milímetros. As mensurações das variáveis massa corporal e estatura foram realizadas conforme Lohman ${ }^{14}$. $\mathrm{O}$ índice de massa corporal (IMC) foi calculado dividindo-se a massa corporal pela estatura ao quadrado $\left(\mathrm{kg} / \mathrm{m}^{2}\right)$.

A composição corporal foi avaliada por meio da absortometria radiológica de dupla energia (DXA). O equipamento utilizado na DXA foi o densitômetro Lunar Prodigy Advance DXA System versão 13.31. O exame foi realizado por um técnico especializado na Divisão de Raio X e Densitometria Óssea da Divisão de Saúde da Universidade Federal de Viçosa.

Após a análise de toda a área corporal, foram determinadas a gordura corporal relativa (\%), a massa de gordura (MG) e a massa livre de gordura (MLG) das seguintes regiões corporais: corpo inteiro, membros superiores, membros inferiores e tronco. Os membros foram isolados do tronco e da cabeça por meio de linhas geradas pelo programa em marcos anatômicos específicos ${ }^{15}$.

As curvas força-tempo foram analisadas por meio de um algoritmo desenvolvido em linguagem Matlab ${ }^{\circledR}$ (Software Matlab R2009a). Inicialmente, as três tentativas realizadas em cada teste foram utilizadas para o teste de confiabilidade, o que garantiu que a curva cuja tentativa atingiu a maior força máxima fosse selecionada e então utilizada para as análises posteriores. A curva selecionada foi suavizada por meio de janelamento (regressão linear ponderada localmente) pelo método dos quadrados mínimos. O tamanho da janela usada para o processo de regressão local foi definido em 10\% do valor da frequência de aquisição dos dados, correspondendo a 100 pontos, referentes a 0,1 segundos da curva.

A posição inicial para os testes de preensão manual e de extensão do joelho originou os valores de pré-carga das curvas força-tempo que se referiram aos valores registrados da força compreendidos entre o ajuste da mão ou perna nos respectivos dinamômetros, até o efetivo esforço (figura 1). Os valores de pré-carga foram considerados adequados ao estudo se inferiores a $5 \mathrm{kgf}$. Portanto, para se identificar o início efetivo do esforço, utilizou-se como critério o valor da força que, ao término da fase de pré-carga, fosse o mínimo de desvios padrões, acima do valor médio desse trecho, necessários para que o início efetivo do esforço fosse determinado. Tal análise foi realizada por meio de inspeção visual da curva de força.

Algumas variáveis da curva força-tempo foram identificadas, tais como a força máxima e o tempo necessário para alcançá-la, bem como

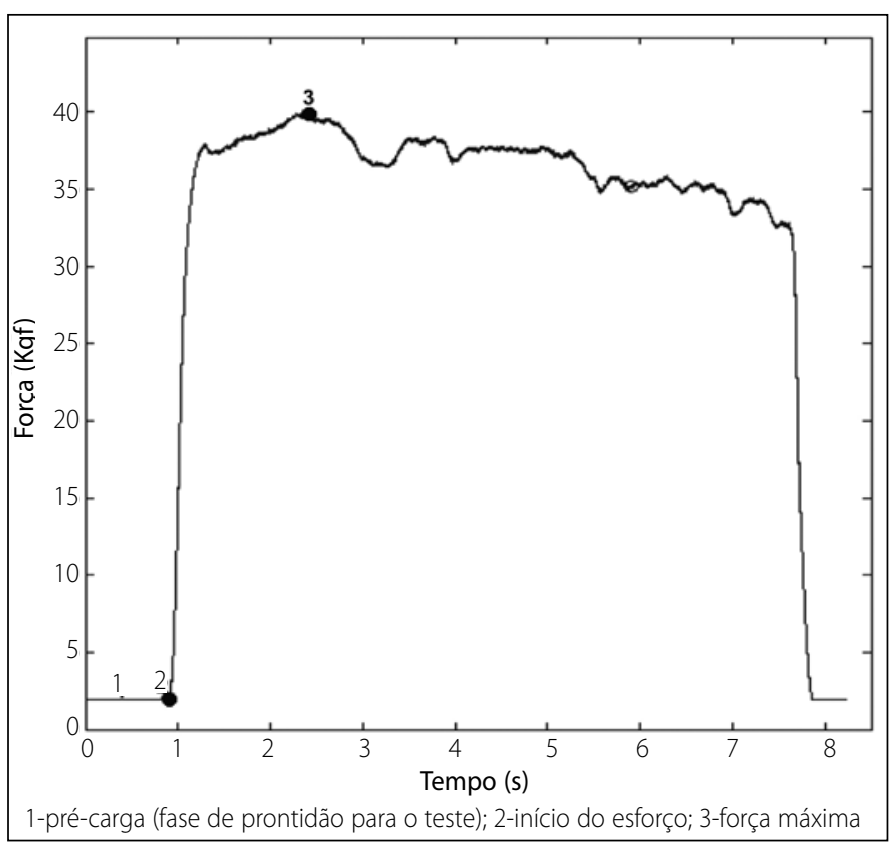

Figura 1. Exemplo de curva típica da força muscular obtida nos testes de esforço isométrico. a taxa de desenvolvimento da força ( $\triangle$ força/ $\triangle$ tempo) obtida para os intervalos de tempo de 0-50; 0-100; 0-150; 0-200 e 0-250ms, referentes ao início da contração.

\section{Análise estatística}

A confiabilidade foi avaliada por meio do coeficiente de correlação intraclasse $(\mathrm{CCl})$ calculado para as três tentativas de cada teste, bem como para o intervalo de confiança de 95\%. Para a avaliação das características descritivas da amostra, foram utilizadas medidas de tendência central e de dispersão (média e desvio padrão, respectivamente). Para verificar a normalidade da distribuição de todas as variáveis analisadas, utilizou-se o teste de Shapiro-Wilk. As possíveis diferenças relacionadas à idade nas variáveis analisadas foram verificadas por meio da análise de variância com os testes ANOVA de um fator (variável) e três níveis (grupos etários), seguida do post hoc de Tukey para as variáveis que apresentaram distribuição normal, e Kruskal-Wallis para as variáveis que violaram esse pressuposto. Para a análise dos dados, foi utilizado o software Statistica (versão 7.0). O nível de significância adotado foi $p<0,05$.

\section{RESULTADOS}

A tabela 1 apresenta a idade e as características de composição corporal das voluntárias divididas entre os grupos JO, MI e ID.

As descrições dos valores de força máxima entre as tentativas, tanto de preensão manual quanto de extensão do joelho estão dispostas na tabela 2, juntamente com o cálculo do coeficiente de correlação intraclasse para as três tentativas e de seus respectivos intervalos de confiança. Os altos coeficientes de correlação intraclasse demonstrado entre as tentativas de força de preensão manual $(0,96)$ e de extensão do joelho $(0,98)$ sugerem grande reprodutibilidade entre as tentativas, e dessa forma permitiu o uso da tentativa de maior força máxima para as análises referentes ao comportamento temporal da força.

Os dados de força máxima $\left(F_{\text {max }}\right)$ e tempo necessário para alcançá-la $\left(T_{-} F_{\text {max }}\right)$, bem como as TDF obtida nos intervalos de tempo de 0-50; 0-100, 0-150; 0-200 e 0-250ms normalizadas pela força máxima estão dispostos nas tabelas 3 e 4 .

A TDF obtida nos intervalos de tempo de 0-50; 0-100, 0-150; 0-200 e 0-250ms para preensão manual e extensão do joelho está representada nas figuras 2 e 3.

Tabela 1. Descrição das características gerais das voluntárias.

\begin{tabular}{c|c|c|c|c}
\hline Características & JO $(\mathbf{n}=\mathbf{2 4})$ & MI $(\mathbf{n}=\mathbf{1 3})$ & ID $(\mathbf{n = 1 5 )}$ & Valor de $\mathbf{p}$ \\
\hline Idade $($ anos) & $25,2 \pm 3,7$ & $58,0 \pm 4,2^{*}$ & $71,3 \pm 3,9^{*}+$ & $<0,000$ \\
\hline Massa corporal $(\mathrm{kg})$ & $59,1 \pm 8,1$ & $66,7 \pm 11,4^{*}$ & $61,6 \pm 5,0$ & 0,019 \\
\hline Estatura $(\mathrm{cm})$ & $161,3 \pm 5,8$ & $153,5 \pm 5,0^{*}$ & $152,8 \pm 5,1^{*}$ & $<0,000$ \\
\hline IMC $\left(\mathrm{kg} / \mathrm{m}^{2}\right)$ & $22,7 \pm 2,9$ & $28,3 \pm 4,5^{*}$ & $26,4 \pm 2,3^{*}$ & 0,000 \\
\hline Gordura corporal $(\%)$ & $30,5 \pm 5,8$ & $38,0 \pm 5,8^{*}$ & $37,8 \pm 4,6^{*}$ & $<0,000$ \\
\hline MLG total $(\mathrm{kg})$ & $39,0 \pm 3,7$ & $39,6 \pm 4,6$ & $37,0 \pm 2,7$ & 0,140 \\
\hline MLG MMSS $(\mathrm{kg})$ & $1,9 \pm 0,3$ & $1,9 \pm 0,3$ & $1,7 \pm 0,2$ & 0,207 \\
\hline MLG MMII $(\mathrm{kg})$ & $7,8 \pm 0,7$ & $7,1 \pm 1,0^{*}$ & $6,4 \pm 0,7^{*}$ & $<0,000$ \\
\hline
\end{tabular}

Média \pm desvio-padrão; $n=$ número de participantes; ${ }^{*}$ vs. grupo JO; † vs. grupo $\mathrm{Ml} ; \mathrm{JO}=$ jovem; $\mathrm{Ml}=$ meia idade; $I D=$ idosas; $I M C=$ índice de massa corporal; $M L G=$ massa livre de gordura; $M M S S=$ membro superior; $M M I I=$ membro inferior.

Tabela 2. Coeficiente de correlação intraclasse (CCI) entre as tentativas de força máxima para ambos os testes ( $n=52)$.

\begin{tabular}{c|c|c}
\hline \multirow{2}{*}{} & \multicolumn{2}{|c}{ Força máxima (kgf) } \\
\cline { 2 - 3 } & Preensão manual & Extensores do joelho \\
\hline Tentativa 1 & $26,1 \pm 4,8$ & $34,4 \pm 9,1$ \\
\hline Tentativa 2 & $26,4 \pm 4,7$ & $36,4 \pm 9,6$ \\
\hline Tentativa 3 & $26,3 \pm 4,7$ & $37,1 \pm 10,6$ \\
\hline CCl & 0,96 & 0,98 \\
\hline IC 95\% & $0,94-0,97$ & $0,96-0,98$ \\
\hline
\end{tabular}

Média \pm desvio-padrão; $C \mathrm{Cl}=$ coeficiente de correlação intraclasse; $\mathrm{IC}=$ intervalo de confiança. 
Tabela 3. Parâmetros do desenvolvimento temporal da força de preensão manual.

\begin{tabular}{c|c|c|c|c}
\hline Variável & JO $(\mathbf{n}=\mathbf{2 4})$ & $\mathbf{M I}(\mathbf{n}=\mathbf{1 3})$ & $\mathbf{I D}(\mathbf{n}=\mathbf{1 5})$ & Valor de $\mathbf{p}$ \\
\hline $\mathrm{F}_{\max }(\mathrm{kgf})$ & $27,9 \pm 4,6$ & $27,2 \pm 4,7$ & $27,0 \pm 4,3$ & 0,831 \\
\hline $\mathrm{T}_{-} \mathrm{F}_{\max }(\mathrm{s})$ & $1,6 \pm 1,2$ & $2,3 \pm 1,0^{*}$ & $2,1 \pm 0,8$ & 0,024 \\
\hline TDFn 0-50 (ms) & $4,4 \pm 3,0$ & $1,1 \pm 0,9^{*}$ & $1,2 \pm 1,2^{*}$ & 0,000 \\
\hline TDFn 0-100 (ms) & $3,7 \pm 1,9$ & $1,5 \pm 0,8^{*}$ & $1,7 \pm 1,3^{*}$ & 0,000 \\
\hline TDFn 0-150 (ms) & $2,3 \pm 1,2$ & $1,8 \pm 1,0$ & $1,6 \pm 1,0$ & 0,187 \\
\hline TDFn 0-200 (ms) & $1,6 \pm 0,9$ & $1,8 \pm 0,8$ & $1,4 \pm 0,7$ & 0,533 \\
\hline TDFn 0-250 (ms) & $1,2 \pm 0,8$ & $1,6 \pm 0,6$ & $1,2 \pm 0,5$ & 0,084
\end{tabular}

Média \pm desvio-padrão; $n=$ número de participantes; * vs. grupo $\mathrm{JO}$; $\mathrm{JO}=$ jovem; $\mathrm{Ml}=$ meia idade; $\mathrm{ID}=$ idosas; Fmax=força máxima; T_Fmax= tempo necessário para atingir a força máxima TDFn= taxa de desenvolvimento da força normalizada pela força máxima.

Tabela 4. Parâmetros do desenvolvimento temporal da força dos extensores do joelho.

\begin{tabular}{c|c|c|c|c}
\hline Variável & JO $(\mathbf{n}=\mathbf{2 4})$ & $\mathbf{M I}(\mathbf{n}=\mathbf{1 3})$ & $\mathbf{I D}(\mathbf{n}=\mathbf{1 5})$ & Valor de $\mathbf{p}$ \\
\hline $\mathrm{F}_{\max }(\mathrm{kgf})$ & $43,5 \pm 10,4$ & $35,6 \pm 7,2^{*}$ & $30,1 \pm 6,1^{*}$ & 0,000 \\
\hline $\mathrm{T}_{-} \mathrm{F}_{\max }(\mathrm{s})$ & $1,7 \pm 1,1$ & $2,3 \pm 1,1$ & $2,4 \pm 0,9$ & 0,067 \\
\hline TDFn 0-50 (ms) & $4,1 \pm 3,7$ & $2,1 \pm 1,8$ & $0,8 \pm 1,0^{*}$ & 0,007 \\
\hline TDFn 0-100 (ms) & $2,5 \pm 1,6$ & $2,1 \pm 1,1$ & $1,4 \pm 1,4$ & 0,051 \\
\hline TDFn 0-150 (ms) & $2,2 \pm 1,3$ & $1,5 \pm 0,8$ & $1,1 \pm 1,0^{*}$ & 0,012 \\
\hline TDFn 0-200 (ms) & $1,9 \pm 1,1$ & $1,5 \pm 0,6$ & $1,2 \pm 0,7^{*}$ & 0,044 \\
\hline TDFn 0-250 (ms) & $1,5 \pm 0,8$ & $1,2 \pm 0,5$ & $1,0 \pm 0,7$ & 0,174 \\
\hline
\end{tabular}

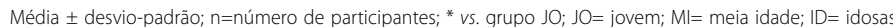
Fmax=força máxima; T_Fmax= tempo necessário para atingir a força máxima; TDFn= taxa de desenvolvimento da força normalizada pela força máxima.

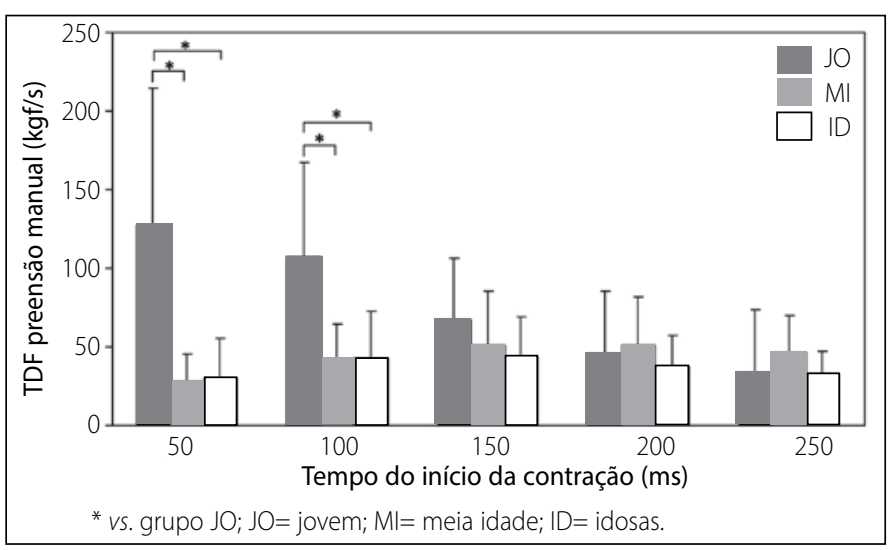

Figura 2. Taxa de desenvolvimento da força de preensão manual.

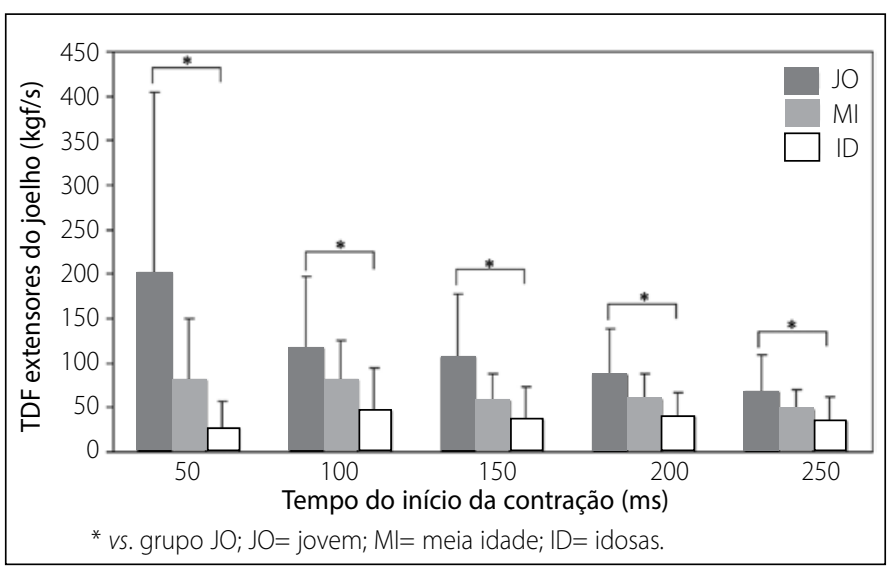

Figura 3. Taxa de desenvolvimento da força dos extensores do joelho.

\section{DISCUSSÃO}

No presente estudo, a MLG do membro superior e a força de preensão manual não evidenciaram alterações significativas entre os grupos. Em contrapartida, um paralelo declínio na MLG e força do membro inferior foi observado nas mulheres de Ml e ID. Esses achados estão em acordo com outros estudos, os quais também observaram reduções mais pronunciadas nos membros inferiores ${ }^{6,16}$. O declínio diferenciado entre os membros tem sido relacionado a alterações nos padrões de atividades físicas realizadas. Ferreira et al. ${ }^{17}$ compararam o nível e a intensidade de atividade dos membros inferiores e superiores de mulheres brasileiras jovens e idosas fisicamente ativas. Seus resultados sugeriram que, com o avançar da idade, o nível de atividade física dos membros superiores aumentam, enquanto que dos membros inferiores declinam. Adicionalmente, Theou et al. ${ }^{18}$ investigaram a atividade muscular diária e a quiescência dos músculos vasto lateral, reto femoral e tríceps e bíceps braquiais em mulheres entre 68 e 90 anos. A atividade eletromiográfica foi registrada durante nove horas e evidenciou maior ativação nos músculos do membro superior em comparação ao membro inferior. Os resultados desses estudos podem ajudar a explicar o declínio mais acentuado na força dos membros inferiores do que dos membros superiores.

As reduções relacionadas à idade na força e potência são associadas ao declínio da massa muscular, que por sua vez, é mediada por redução no tamanho e/ou no número de fibras musculares individuais, especialmente as de contração rápida ${ }^{19}$. Contudo, redução na força específica (força/unidade de área muscular), lentidão na velocidade de encurtamento, reduzida condução neural para o músculo e aumento da rigidez músculo-tendínea também podem afetar a performance muscular do idoso ${ }^{20,21}$.

Decréscimo na TDF dos idosos tem sido observado em prévias investigações ${ }^{3,8,16}$. Neste estudo, a TDF de preensão manual evidenciou valores inferiores nos grupos Ml e ID em comparação ao grupo JO no início da contração muscular (50 e 100ms). Enquanto no estudo de Watanabe et al. ${ }^{3}$ valores inferiores de TDF foram observados em todos os intervalos de tempo (0-250ms), a exceção do intervalo entre 0 e $20 \mathrm{~ms}$. A idade mais avançada das mulheres que compuseram o grupo de idosas no estudo de Watanabe et al. ${ }^{3}$ (70-92 anos) pode estar relacionada às diferenças entre os resultados apresentados. Do mesmo modo, diferenças na qualidade e quantidade das atividades físicas realizadas pelas voluntárias também podem justificar tais discrepâncias. Adicionalmente, estudos têm sugerido que o período inicial da contração muscular (30-100ms) seja mais influenciado por características de ativação neuromuscular ${ }^{4} \mathrm{e}$ pela rigidez dos elementos elásticos em série ${ }^{22,23}$ do que os intervalos finais que parecem mais relacionados à força e, indiretamente, à massa muscular ${ }^{10,24}$. Assim, é possível que os grupos MI e ID analisadas neste estudo exibam um aumento na rigidez músculo-tendínea e/ou alterações na ativação neuromuscular no membro superior, o que, por sua vez, justificaria tais alterações na fase inicial da curva força-tempo. Do mesmo modo, o fato de os grupos MI e ID não apresentarem alterações na força e na massa muscular justificaria a manutenção da TDF nos intervalos finais da curva de força.

Já a TDF dos extensores do joelho demonstrou-se preservada no grupo MI, porém, reduzida no grupo ID em comparação ao JO, em todos os intervalos analisados. Thompson et al. ${ }^{4}$ encontraram TDF preservada em homens de meia idade em comparação aos jovens, sugerindo que o declínio na TDF dos extensores do joelho se inicie a partir da quarta década. Nesta investigação, o declínio na TDF das idosas em todos os intervalos de tempo analisados sugere que o membro inferior possa ser influenciado por diversos fatores fisiológicos, tais como prejuízos na ativação neural e aumento da rigidez músculo-tendínea, além da redução da massa muscular e força máxima conforme constatado. Ademais, segundo Pereira e Gonçalves ${ }^{25}$, o aumento da coativação da musculatura antagonista também está envolvido na redução da TDF dos extensores do joelho em mulheres idosas.

Quando normalizados pela força máxima, os intervalos da TDF de preensão manual, demonstraram comportamento similar o que fortalece a conjectura de que as alterações relacionadas à idade sobre a TDF de preensão manual não estão atreladas apenas às alterações na força e, indiretamente, à MLG com o envelhecimento, mas também a prejuízos 
nas propriedades intrínsecas da musculatura. A TDF dos extensores do joelho também demonstrou comportamento similar aos valores absolutos, à exceção dos trechos 0-100 e 0-250, o que sugere que a redução na força exercida pelas voluntárias possa, pelo menos nesses trechos, exercer um importante papel na redução da TDF dos membros inferiores.

Segundo Petrella et al. ${ }^{26}$, a redução da TDF decorrente do processo de envelhecimento tem sido atribuída a diferentes fatores. Dentre eles, a redução da massa muscular e a atrofia das fibras de contração rápida têm sido apontadas como fatores primários. No entanto, prejuízos na atividade da miosina ATPase e/ou no acoplamento excitação-contração ${ }^{27}$ também têm sido apontados como fatores relacionados à lentidão muscular consequente ao envelhecimento. As mudanças nas propriedades intrínsecas dos músculos podem ser acompanhadas por prejuízo na ativação voluntária o que também reduz a TDF. A modulação da força durante uma contração voluntária depende do número e do tipo de unidades motoras recrutadas, bem como da taxa na qual essas unidades geram potenciais de ação ${ }^{28}$. Klass et al. ${ }^{29}$ investigaram a associação entre a taxa de desenvolvimento do torque e a máxima frequência de disparo das unidades motoras em jovens e idosos durante ação isométrica submáxima do músculo tibial anterior. Para esses autores, não apenas a lentidão das propriedades contráteis do músculo, mas também a frequência de disparo das unidades motoras limitam a máxima taxa de desenvolvimento de torque dos dorsiflexores. Em contrapartida, Jesunathadas et al. ${ }^{30}$, ao analisarem as características de recrutamento e desrecrutamento das unidades motoras do primeiro interósseo dorsal, não encontraram diferenças entre jovens e idosos na frequência de disparo das unidades motoras. Esses estudos demonstram que as alterações neurais também parecem ocorrer de modo diferente de acordo com o grupamento muscular analisado e, desse modo, podem representar diferentes papéis na redução da TDF com idade.

Neste estudo, o tempo necessário para atingir a força máxima de preensão manual e dos extensores do joelho foi superior nos grupos Ml e ID em relação ao grupo JO, embora redução significativa tenha sido observada apenas no grupo MI durante o teste de preensão manual. Esse

\section{REFERÊNCIAS}

1. Klass M, Baudry S, Duchateau J. Voluntary activation during maximal contraction with advancing age: a brief review. Eur J Appl Physiol. 2007;100(5):543-51.

2. Pääsuke M, Ereline J, Gapeyeva H, Sirkel S, Sander P. Age-related differences in twitch contractile properties of plantarflexor muscles in women. Acta Physiol Scand. 2000;170(1): 51-7.

3. Watanabe K, Tsubota S, Chin G, Aoki M. Differences in Parameters of the Explosive Grip Force Test Between Young and Older Women. J Gerontol A Biol Sci Med Sci. 2011;66(5): 554-8.

4. Thompson BJ, Ryan ED, Sobolewski EJ, Conchola EC, Cramer JT. Age related differences in maximal and rapid torque characteristics of leg extensors and flexors in young, midle-aged and men. Exp Gerontol. 2013;48(2):277-82.

5. Aagaard P, Simonsen EB, Andersen JL, Magnusson P, Dyhre-Poulsen P. Increased rate of force development and neural drive of human skeletal muscle following resistance training. J Appl Physiol. 2002;93(4):1318-26.

6. Candow DG, Chilibeck PD. Differences in size, strength, and power of upper and lower body muscle groups in young and older men. J Gerontol A Biol Sci Med Sci 2005;60(2):148-56.

7. Lynch NA, Metter EJ, Lindle RS, Fozard JL, Tobin JD, Roy TA, et al. Muscle quality. I. Age-associated differences between arm and leg muscle groups. J Appl Physiol. 1999;86(1):188-94.

8. Aoki H, Demura S. Age differences in hand grip power in the elderly. Arch Gerontol Geriatr. 2011;52(3):176-9.

9. Ditroilo M, Fortes R, Benelli P, Gambarara D, Vito G. Effect of age and limb dominance on upper and lower limb muscle function in healthy males and females aged 40-80 years. J Sports Sci. 2010;28(6):667-77.

10. Andersen $L$, Aagaard P. Influence of maximal muscle strength and intrinsic muscle contractile properties on contractile rate of force development. Eur J Appl Physiol. 2006;96(1):46-52.

11. Novo Júnior J, inventor; Universidade Federal de Juiz de Fora, cessionário. Configuração aplicada em empunhadura. Brasil patente DI 6901797-2. 2009 mai 05.

12. Fess E. Grip strength. In: Casanova JS.Clinical assessment recommendations. 2nd. ed. Chicago: American Society of Hand Therapists; 1992. p. 41-5.

13. Amaral JF, Mancini M, Novo Júnior JM. Comparação de três dinamômetros de preensão manual relacionados à exatidão e precisão das medidas. Rev Bras Fisioter. 2012;16 (3):216-24.

14. Lohman TG. Advances in body composition assessment. Champaign, LL: Human Kinetics Publishers; 1992.

15. Heymsfield SB, Smith R, Aulet M, Bensen B, Lichtman S, Wang J, et al. Appendicular skeletal muscle mass: measurement by dual-photon absorptiometry. Am J Clin Nutr. 1990;52(2):214-8. incremento no tempo de desenvolvimento da força máxima demonstra uma reduzida capacidade de gerar força das mulheres de meia idade e idosas em comparação às jovens. Não foram encontradas investigações com o propósito de analisar o referido tempo em mulheres, sejam elas jovens ou idosas, o que dificulta a comparação dos achados deste estudo.

Tendo em vista que o declínio da capacidade de desenvolver força rápida durante o envelhecimento está relacionado ao aumento da debilidade funcional e ao risco de lesões em atividades comuns da vida diária em idosos ${ }^{4}$, ressalta-se a importância destes resultados, que, embora não possam ser extrapolados para força muscular de testes dinâmicos, uma vez que foram realizados em condições isométricas, demonstram a importância de se definir uma estratégia de treinamento adequado para mulheres idosas e de meia idade, sobretudo dos membros inferiores, de forma a possibilitar uma manutenção da funcionalidade e qualidade de vida dessa população. Este estudo tem limitação inerente à natureza transversal da pesquisa. Dessa forma, investigações longitudinais são necessárias para confirmar estes achados.

\section{CONCLUSÃO}

Em síntese, os resultados deste estudo sugerem que os grupos musculares dos membros superiores e inferiores possam ser afetados pelo envelhecimento em diferentes magnitudes e, possivelmente, em resposta a diferentes fatores. As diferenças entre os membros podem estar relacionadas à quantidade e qualidade das atividades físicas realizadas.

\section{AGRADECIMENTOS}

Aos alunos da UFJF Marcelly Mancini, Letícia Moreira, Josiane Almeida, Marcos Paulo Azevedo e Fred Frizero pelo auxílio nas coletas de dados desta pesquisa. À Coordenação de Aperfeiçoamento de Pessoal de Nível Superior (CAPES) pelo incentivo financeiro.

Todos os autores declararam não haver qualquer potencial conflito de interesses referente a este artigo.

16. Izquierdo M, Ibañez J, Gorostiaga E, Garrues M, Zúñiga A, Antón A, et al. Maximal strenght and power characteristics in isometric and dynamic actions of the upper and lower extremities in middle-age and older men. Acta Physiol Scand. 1999;167:57-68.

17. Ferreira L, Gobbi S, Gobbi LT. An explanatory mechanism for the different decline in limb strength in older women. Arch Gerontol Geriatr. 2009;49(3):373-7.

18. Theou $\mathrm{O}$, Jones GR, Vandervoort AA, Jakobi JM. Daily muscle activity and quiescence in non-frail, pre-frail, and frail older women. Exp Gerontol. 2010;45(12):909-17.

19. Thompson LV. Effect of age and training on skeletal muscle physiology and performance. Phys Ther. 1994;74(1):71-81

20. D'Antona G, Pellegrino MA, Carlizzi CN, Bottinelli R. Deterioration of contractile properties of muscle fibers in elderly subjects is modulated by the level of physical activity. Eur J Appl Physiol. 2007;100(5):603-11.

21. Magnusson SP, Simonsen EB, Aagaard P, Boesen J, Johannsen F, Kjaer M. Determinants of musculoskeletal flexibility: viscoelastic properties, cross-sectional area, EMG and stretch tolerance. Scand I Med Sci Sports. 1997;7(4):195-202.

22. Edman KA, Josephson RK. Determinants of force rise time during isometric contraction of frog muscle fibres. J Physiol. 2007;580(3):1007-19.

23. Blazevich AJ, Cannavan D, Horne S, Coleman DR, Aagaard P. Changes in muscle force-length properties affect the early rise of force in vivo. Muscle Nerve. 2009;39(4):512-20.

24. Mirkov DM, Nedeljkovic A, Milanovic S, Jaric S. Muscle strength testing: evaluation of tests of explosive force production. Eur J Appl Physiol. 2004;91(2-3):147-54.

25. Pereira MP, Gonçalves M. Muscular coativaction (CA) around the knee reduces power production in elderly women. Arch Gerontol Geriatr. 2011;52(3):317-21.

26. Petrella JK, Kim JS, Tuggle SC, Hall SR, Bamman MM. Age differences in knee extension power, contractile velocity, and fatigability. J Appl Physiol. 2005; 98(1):211-20.

27. Zhong S, Chen CN, Thompson LV. Sarcopenia of ageing: functional, structural and biochemical alterations. Rev Bras Fisioter. 2007;11(2):91-7.

28. Clamann HP. Motor unit recruitment and the gradation muscle force. Phys Ther. 1993;73(12):830-43.

29. Klass M, Baudry S, Duchateau J. Age-related decline in rate of torque development is accompanied dy lower maximal motor unit discharge frequency during fast contractions. J Appl Physiol. 2008;104(3):739-46.

30. Jesunathadas M, Marmon AR, Gibb JM, Enoka RM. Recruitment and derecruitment characteristics of motor units in a hand muscle of young and adults. J Appl Physiol 2010;108(6):1659-67. 